\title{
BMJ Open Interventions to improve screening and appropriate referral of patients with cancer for distress: systematic review protocol
}

\author{
Kristen McCarter, ${ }^{1}$ Ben Britton, ${ }^{2}$ Amanda Baker, ${ }^{2}$ Sean Halpin, ${ }^{1}$ Alison Beck, ${ }^{2}$ \\ Gregory Carter, ${ }^{2}$ Chris Wratten, ${ }^{3}$ Judy Bauer, ${ }^{4}$ Debbie Booth, ${ }^{5}$ Erin Forbes, ${ }^{2}$ \\ Luke Wolfenden ${ }^{6}$
}

To cite: McCarter $\mathrm{K}$, Britton B, Baker A, et al. Interventions to improve screening and appropriate referral of patients with cancer for distress: systematic review protocol. BMJ Open 2015;5:e008277. doi:10.1136/bmjopen-2015008277

- Prepublication history and additional material is available. To view please visit the journal (http://dx.doi.org/ 10.1136/bmjopen-2015008277)

Received 24 March 2015 Revised 7 July 2015 Accepted 4 August 2015

CrossMark

For numbered affiliations see end of article.

Correspondence to Kristen McCarter; Kristen. McCarter@newcastle.edu.au

\section{ABSTRACT}

Introduction: It is estimated that $35-40 \%$ of patients with cancer experience distress at some stage during their illness. Distress may affect functioning, capacity to cope, treatment compliance, quality of life and survival of patients with cancer. Best practice clinical guidelines recommend routine psychosocial distress screening and referral for further assessment and/or psychosocial support for patients with cancer. However, evidence suggests this care is not provided consistently.

Methods and analysis: We developed our methods following the Preferred Reporting Items for Systematic Reviews and Meta-Analysis (PRISMA) statement. The review is registered with PROSPERO and any amendments to the protocol will be tracked. The primary aim of this systematic review is to examine the impact of interventions delivered in healthcare settings that are aimed at (1) improving routine screening of patients for psychosocial distress and (2) referral of distressed patients with cancer for further assessment and/or psychosocial support. The effectiveness of such interventions in reducing psychosocial distress, and any unintended adverse effect of the intervention will also be assessed in patients with cancer. Data sources will include the bibliographic databases Cochrane Central Register of Controlled trials (CENTRAL) in the Cochrane Library, MEDLINE, EMBASE, PsycINFO and CINAHL. Eligible studies must compare an intervention (or two or more interventions) in a healthcare setting to improve the rate of screening for psychosocial distress and/or referral for further assessment and/or psychosocial support for patients with cancer with no intervention or 'usual' practice. Two investigators will independently review titles and abstracts, followed by full article reviews and data extraction. Disagreements will be resolved by consensus and if necessary, a third reviewer. Where studies are sufficiently homogenous, trial data will be pooled and metaanalyses performed.

Ethics and dissemination: No ethical issues are foreseen. The findings of this study will be disseminated widely via peer-reviewed publications and conference presentations.
Systematic review registration: PROSPERO

registration number CRD4 2015017518.

\section{INTRODUCTION}

Rationale

Between $35 \%$ and $40 \%$ of patients with cancer experience distress at some stage during their illness. ${ }^{1}$ Despite this, distress is often unrecognised in patients with cancer by clinicians. ${ }^{2}$ Psychological distress can arise in response to cancer-related factors such as diagnosis and cancer progression. ${ }^{2}$ Distress may affect functioning, capacity to cope, treatment compliance, quality of life and survival of patients with cancer, ${ }^{13}$ and increase the treatment burden to the medical team and healthcare system. ${ }^{4}$ Addressing distress in cancer populations is, therefore, an important health priority.

The importance of psychosocial care for patients with cancer is recognised by professional associations and is included in clinical guidelines. ${ }^{5}{ }^{6}$ The National Comprehensive Cancer Network Clinical Practice Guidelines in Oncology: Distress Management, ${ }^{1}$ and the National Institute for Clinical Excellence guidance manual, Improving Supportive and Palliative Care for Adults with Cancer ${ }^{7}$ recommend routine screening for psychosocial distress and subsequent assessment or referral to appropriate services by those responsible for the care of patients with cancer. The Institute of Medicine report, Care for the Whole Patient recommends screening for distress and the development of a treatment plan with referrals as needed to psychosocial services. In 2015, the American College of Surgeons Commission on Cancer will require cancer centres to implement screening programmes for psychosocial distress as a new criterion for 
accreditation. ${ }^{9}$ Systematic reviews and meta-analyses on which these recommendations are based have demonstrated distress screening and referral improves the identification and management of psychosocial distress and reduces psychological morbidity in patients with cancer. ${ }^{3} 10$

Despite evidence-based guideline recommendations, screening and referral of patients with cancer for psychosocial distress is not routinely conducted by clinicians responsible for the clinical management of patients with cancer. ${ }^{11}$ While previous reviews of interventions have examined the effects of common distress screening tools, for example, the Distress Thermometer ${ }^{1}$ on patients with cancer outcomes such as quality of life or depression, ${ }^{12-16}$ or the impact of patient-reported outcome measures to improve identification of distressed patients and improve treatment decisions, ${ }^{17} 18$ we are not aware of any previous systematic review of interventions to improve clinician provision of screening and appropriate referral of patients with cancer per se. Reviews of clinical practice changes in interventions more broadly suggest that a range of interventions may be effective in improving clinicians' provision of care consistent with guidelines recommendations such as educational strategies, audit and feedback, use of reminders and multiprofessional collaboration. ${ }^{19-21}$

\section{Objectives}

In the absence of reviews particularly aimed at interventions to increase screening and referral for distress in patients with cancer, the primary aims of this review are to determine the impact of interventions to improve clinician provision of screening and appropriate referral of patients with cancer for distress. In particular, we will assess the impact of such interventions on:

1. Improving screening of patients for psychosocial distress;

2. Improving referral of patients with cancer who screen positive on a measure of distress for further assessment and/or psychosocial support.

The secondary aims of the review are to:

1. Describe the effectiveness of such interventions on reducing psychosocial distress of patients with cancer;

2. Describe any unintended adverse effects of such an intervention.

\section{METHODS AND ANALYSIS}

The review methods are based on the Preferred Reporting Items for Systematic Reviews and Meta-Analysis Statement. ${ }^{22}$

\section{Eligibility criteria}

Study characteristics

\section{Types of studies}

Inclusion criteria

Studies with the following study designs will be included:

- Randomised controlled trials, including cluster randomised controlled trials;

- Staggered enrolment trials or stepped-wedged trials;
- Quasi-randomised trials;

- Quasi-experimental trials with comparison/control groups, including non-randomised pre-post (beforeafter) trials with one or more intervention and control groups, time-series/interrupted time-series trials (including multiple baseline trials) with independent control groups, preference trials and regression discontinuity trials;

- Natural experiment studies that have a comparison group.

Any trial without parallel comparison or control groups will be excluded. There will be no restriction based on length of follow-up. There will be no restrictions based on the year of the study publication or language. Only studies published in peer-reviewed scientific journals will be included.

\section{Participants}

Inclusion criteria

Participants could include:

1. Adult patients with cancer who are about to undergo, are currently undergoing or have undergone medical treatment, including radiotherapy, chemotherapy, surgery or combined modality;

2. Clinical staff members such as physicians, surgeons and oncologists, nurses, and allied health professionals responsible for the care of patients with cancer at any stage of treatment within primary and secondary healthcare settings such as hospitals, general practices or oncology clinics;

3. Administrative staff of health services including hospital managers and quality assurance staff responsible for improving the delivery of health services to patients with cancer; government or non-government cancer services or other organisations that may influence screening and referral of patients with cancer.

\section{Exclusion criteria}

Studies which examine screening for psychosocial distress and/or referral for appropriate psychosocial support for carers of patients with cancer or survivors of cancer will be excluded. Studies reporting on patients with cancer under the age of 18 will be excluded.

\section{Types of interventions} Inclusion criteria

Interventions will be included that are implemented in a health setting that aim to improve the rate of routine screening procedures for psychosocial distress and/or referral for appropriate psychosocial support in healthcare settings. Interventions could include quality improvement initiatives, education and training, ${ }^{23-25}$ performance feedback, prompts and reminders ${ }^{19}$ implementation resources, ${ }^{26}$ financial incentives ${ }^{27}$ or the use of opinion leaders. ${ }^{23}{ }^{28}$ Interventions could be singular or multicomponent.

Consistent with the definition of distress provided by the National Cancer Network, ${ }^{1}$ psychosocial distress will 
include any form of experienced distress, which may be due to emotional, psychological, social or spiritual factors. For the purposes of the review, distress screening is defined as the standardised brief assessment of patients to determine whether referral for more extensive assessment and/or psychosocial support services is warranted. Trials of interventions to improve the use of standardised screening tools or instruments with or without additional clinical judgement will be included. Studies using clinical judgement of psychosocial distress alone, without use of a formal screening tool, will be excluded. Screening instruments could include traditional measures of psychosocial distress such as the Distress Thermometer, ${ }^{1}$ patient-reported outcome measures of psychological distress including depression and anxiety, for example, the Hospital Anxiety and Depression Scale, ${ }^{29}$ and measures of health-related quality of life (HRQoL) that include a psychological distress component as a core component domain, for example, the MOS 36-Item Short-Form Health Survey. ${ }^{30}$ Administration of the screening instrument may be completed orally or via a paper-based questionnaire or computer/tablet questionnaire.

Referral for psychosocial support will include any written or verbal offer or direction of a patient for further review, consultation, assessment or treatment with any health professional, including the primary oncology team or health service offering psychosocial support such as psychooncology services. Referral must be made as part of the implemented intervention and based on the results of a distress-screening instrument. The referral should not be based on clinical judgement alone.

Studies will be included if these implement either distress screening only or distress screening and appropriate referral. Interventions targeting a range of clinical practices, such as treatment or management decisions or medication prescriptions that also include screening for psychosocial distress and/or referral for appropriate psychosocial support will be included only when data for changes in screening and/or referral is reported separately from other outcomes. Studies where research staff conduct the screening or referral will be excluded, as will trials of population-based community screening programmes.

\section{Exclusion criteria}

Studies using clinical judgement of psychosocial distress alone, without use of a formal screening tool, will be excluded. Studies where research staff conduct the screening or referral will be excluded, as will trial of population-based community screening programmes.

\section{Comparisons}

Comparisons will be included that are non intervention controls, 'usual' practice or that are alternative interventions.

\section{Outcomes}

Primary outcomes:

1. Any outcome measure reporting the provision of screening for psychosocial distress will be included (eg, number or per cent of patients with cancer screened); such data may be obtained from medical record audits, client or clinician report, administrative data, audio recording or other sources;

2. And/or any outcome measure of the provision of referral for further assessment and/or psychosocial support (eg, number or per cent of patients with cancer referred); such data may be obtained from medical record audits, client or clinician report, administrative data, audio recording or other sources such as records of referral service use by organisations providing psychosocial care for patients with cancer.

Secondary outcomes:

1. Any validated outcome measure of psychosocial distress in the patients (eg, distress outcome assessments such as the Kessler Psychological Distress Scale) will be included $;{ }^{31}$

2. Any outcome measure of unintended adverse effects or barriers of the intervention to patients, clinicians or health services such as stress in health professionals providing psychosocial screening and referral. ${ }^{32}$

\section{Information sources}

\section{Electronic databases}

The following electronic databases will be searched for potentially eligible studies; the Cochrane Central Register of Controlled trials (CENTRAL) in the Cochrane Library, MEDLINE, EMBASE, PsycINFO and CINAHL. The MEDLINE search strategy below will be adapted for other databases and will include filters used in other systematic reviews for population (patients with cancer), ${ }^{33}$ screening for distress ${ }^{34}$ and referral, ${ }^{35}$ and psychosocial support. ${ }^{36}$

\section{Other sources}

Studies will also be obtained from the following sources:

- Reference lists of included studies;

- Manual searching of three relevant journals in the field (published in the past 5 years): Journal of the National Comprehensive Cancer Network, Psychooncology and Supportive Care in Cancer;

- Manual searching of conference abstracts published in the preceding 2 years from the International Psycho-Oncology Society and the Society of Behavioural Medicine;

- A grey literature search using Google Scholar (published online in the past 5 years-the first 200 citations will be examined).

\section{Search strategy}

The search strategy for MEDLINE is in online supplementary appendix 1 . This strategy will be adapted to the 
other electronic databases, with any modifications reported in the review manuscript.

\section{Study selection}

The titles and abstracts retrieved by electronic searches will be exported to a reference management database (Endnote version $\mathrm{X} 6$ ) to remove duplicates. Two reviewers will independently screen abstracts and titles. The reviewers will not be blind to the author or journal information. Screening of studies will be conducted using a standardised screening tool and will be pilot tested with a sample of articles before use. The abstracts of papers that are in a language other than English will be translated using Google Translate. If considered eligible or eligibility is unclear, professional translation of the full paper will be undertaken.

The full texts of manuscripts will be obtained for all potentially eligible trials for further examination. For all manuscripts, the primary reason for exclusion will be recorded and documented in the excluded studies table. Discrepancies between the two review authors regarding study eligibility will be resolved by discussion and consensus and if necessary, by a third reviewer.

\section{Data extraction}

The two review authors will independently extract data from the included trials using a prepiloted data extraction form that will be developed based on recommendations from the Cochrane Handbook for Systematic Reviews of Interventions. ${ }^{37}$ The data extraction form will be piloted before use. Discrepancies between reviewers regarding data extraction will be resolved by discussion and consensus, and if necessary, include a third reviewer. Information will be transferred from data extraction forms into statistical software for meta-analyses.

\section{Data items}

The following information will be extracted:

- Authors, year and journal;

- Study eligibility, study design, healthcare provider type (eg, nurses), country, healthcare setting (eg, oncology clinic);

- Patient characteristics and demographics, including cancer site, cancer stage, age, sex, cancer treatment type, treatment status (pre/undergoing/post);

- Characteristics of the intervention, including the duration, intervention strategies, the theoretical underpinning of the study (if noted in the study), screening instrument;

- Trial primary and secondary outcomes, including sample size, the data collection method, validity of measures used, any measures of client uptake or use of psychosocial support services following referral, effect size, measures of change in psychosocial distress;

- Source(s) of research funding and potential conflicts of interest;
- Number of participants per experimental condition as well as information to allow assessment of risk of study bias.

Attempts will be made to contact the corresponding authors of included trials in instances where data is unavailable in the published manuscript.

\section{Assessment of risk of bias}

Two review authors will independently assess the risk of bias of all included trials in accordance with the Cochrane Collaboration's tool in the Cochrane Handbook for Systematic Review of Interventions. ${ }^{37}$ Disagreement between raters will be resolved by discussion and consensus with the involvement (if necessary) of a third review author. An additional criterion 'potential confounding' will be included for the assessment of the risk of bias in non-randomised trial designs. ${ }^{37}$

\section{Data analysis}

\section{Summary measures}

There are a variety of commonly used screening instruments and scoring thresholds for psychosocial distress. ${ }^{34}$ As such, it is anticipated that there will be a range of different outcome measures reported across included studies, which may make meta-analysis of the data from these trials inappropriate, in which case the findings of included studies will be presented narratively. However, for the primary outcomes pertaining to provision of screening for distress and referral for further assessment and/or psychosocial care, and secondary outcomes, attempts will be made to conduct meta-analysis using data from included trials. For binary outcomes, the standard estimation of the OR and a 95\% CI will be calculated. For continuous data, the mean difference will be calculated where a consistent measure of outcome is used in included trials. Where different continuous measures are used to examine an outcome, the appropriateness of calculating a standardised mean difference will be considered. Authors of included trials will be contacted to provide additional information if any outcome data is unclear or missing.

\section{Data synthesis and analysis}

Meta-analysis will be performed using random effects models where suitable data and homogeneity exist $\left(\mathrm{I}^{2}\right.$ $<75 \%$ ). Clustered trials will be examined for unit of analysis errors. An effective sample size will be calculated for use in meta-analysis for trials with unit of analysis errors without appropriate statistical adjustment. Data will not be pooled for trials of different study designs (e.g, randomised and non-randomised designs). Sensitivity analysis will be performed by removing studies with a high risk of bias and by removing outliers contributing to statistical heterogeneity.

\section{Assessment of study heterogeneity}

Heterogeneity will be examined using visual inspection of box plots, forest plots and using the $\mathrm{I}^{2}$ statistic. Where 
there is evidence of high heterogeneity $\left(\mathrm{I}^{2}>75 \%\right)$, heterogeneity will be explored via subgroup analyses according to trial intervention and population characteristics. Funnel plots will be generated by statistical software to enable the assessment of publication bias.

\section{Grading the strength of evidence}

As recommended by the Cochrane Handbook for Systematic Reviews of Interventions, ${ }^{37}$ the overall quality of evidence on outcomes will be presented using the GRADE (Grades of Recommendation, Assessment, Development and Evaluation) approach, which involves consideration of within-study risk of bias (methodological quality), directness of evidence, heterogeneity, precision of effect estimates and risk of publication bias. The overall quality of evidence will be rated at four levels: high, moderate, low and very low.

\section{ETHICS AND DISSEMINATION}

The findings of this study will be disseminated via peerreviewed publications and conference presentations. As no primary data collection will be undertaken, no additional formal ethical assessment and informed consent are required.

\section{DISCUSSION}

Despite guideline recommendations for psychosocial distress screening and referral in cancer, research suggests this care is not provided consistently. ${ }^{2}{ }^{38}$ Presently, the effectiveness of interventions aimed at improving clinicians' provision of routine screening and referral for further assessment and/or treatment for psychosocial distress in patients with cancer remains unclear. The conclusions drawn from the present review when disseminated to policymakers, healthcare providers, and researchers will be helpful in identifying effective approaches for designing interventions aimed to improve the rate of routine provision of this cancer care.

\author{
Author affiliations \\ ${ }^{1}$ School of Psychology, The University of Newcastle, Callaghan, New South \\ Wales, Australia \\ ${ }^{2}$ Faculty of Health and Medicine, Centre for Translational Neuroscience and \\ Mental Health, The University of Newcastle, Callaghan, New South Wales, \\ Australia \\ ${ }^{3}$ Department of Radiation Oncology, Calvary Mater Newcastle Hospital, \\ Waratah, New South Wales, Australia \\ ${ }^{4}$ Centre for Dietetics Research, The University of Queensland, St Lucia, \\ Queensland, Australia \\ ${ }^{5}$ University Library, University of Newcastle, Callaghan, New South Wales, \\ Australia \\ ${ }^{6}$ School of Medicine \& Public Health, The University of Newcastle, Callaghan, \\ New South Wales, Australia
}

Contributors KMC is the lead and the guarantor of this review. KMC and LW conceptualised the review and drafted the manuscript. KMC, $L W, B B, A B, S H$, $A B, G C, C W, J B, D B$ and $E F$ revised the protocol critically. DB and KMC developed the search strategy included in the protocol. All authors approved the final version and agree to be accountable for all aspects of the work.

Funding This research received no specific grant from any funding agency in the public, commercial or not-for-profit sectors.
Competing interests None declared.

Provenance and peer review Not commissioned; externally peer reviewed.

Open Access This is an Open Access article distributed in accordance with the Creative Commons Attribution Non Commercial (CC BY-NC 4.0) license, which permits others to distribute, remix, adapt, build upon this work noncommercially, and license their derivative works on different terms, provided the original work is properly cited and the use is non-commercial. See: http:// creativecommons.org/licenses/by-nc/4.0/

\section{REFERENCES}

1. National Comprehensive Cancer Network. NCCN Clinical Practice Guidelines in Oncology: distress management, 2015. http://www. nccn.org/professionals/physician_gls/pdf/distress.pdf

2. Ma L, Pulin P, Feldstain A, et al. The association between malnutrition and psychological distress in patients with advanced head-and-neck cancer. Curr Oncol 2013;20:e554-60.

3. Holland JC, Alici Y. Management of distress in cancer patients. $J$ Support Oncol 2010;8:4-12.

4. Bultz BD, Carlson LE. Emotional distress: the sixth vital sign-future directions in cancer care. Psychooncology 2006;15:93-5.

5. Howell D, Keller-Olaman S, Oliver T, et al. A Pan-Canadian practice guideline: screening, assessment and care of psychosocial distress (depression, anxiety) in adults with cancer. Toronto: Canadian Partnership Against Cancer (Cancer Journey Action Group) and the Canadian Association of Psychosocial Oncology, 2010.

6. Li M, Kennedy EB, Byrne N, et al. The management of depression in patients with cancer: guideline recommendations: cancer care. Ontario: Cancer Care Ontario, 2015.

7. National Institute for Clinical Excellence. Improving Supportive and Palliative Care for Adults with Cancer London: NICE, 2004.

8. Adler N, Page N. Cancer care for the whole patient: meeting psychosocial health needs. Institute of Medicine (IOM), 2008.

9. American College of Surgeons Commission on Cancer. Cancer Program Standards 2012 Version 1.2.1: ensuring patient-centered care, 2012. https://www.facs.org/quality-programs/cancer/coc/ standards

10. Jacobsen $\mathrm{P}$, Donovan K, Swaine Z, et al. Management of anxiety and depression in adult cancer patients: toward an evidence-based approach. In: Chang A, Ganz P, Hayes D, et al., eds. Oncology: an evidence-based approach. New York: Springer-Verlag, 2006:1552-79.

11. Jacobsen PB, Ransom S. Implementation of NCCN distress management guidelines by member institutions. J Natl Compr Canc Netw 2007;5:99-103.

12. Mitchell AJ. Screening for cancer-related distress: when is implementation successful and when is it unsuccessful? Acta Oncologica 2013;52:216-24.

13. Carlson LE, Bultz BD. Cancer distress screening: needs, models, and methods. J Psychosom Res 2003;55:403-9.

14. Bidstrup PE, Johansen C, Mitchell AJ. Screening for cancer-related distress: summary of evidence from tools to programmes. Acta Oncologica 2011;50:194-204.

15. Meijer A, Roseman M, Delisle VC, et al. Effects of screening for psychological distress on patient outcomes in cancer: a systematic review. J Psychosom Res 2013;75:1-17.

16. Carlson LE, Waller A, Mitchell AJ. Screening for distress and unmet needs in patients with cancer: review and recommendations. $J$ Clin Oncol 2012;30:1160-77.

17. Howell D, Molloy S, Wilkinson K, et al. Patient-reported outcomes in routine cancer clinical practice: a scoping review of use, impact on health outcomes, and implementation factors. Ann Oncol 2015;26:1846-58.

18. Chen J, Ou L, Hollis S. A systematic review of the impact of routine collection of patient reported outcome measures on patients, providers and health organisations in an oncologic setting. BMC Health Serv Res 2013;13:211.

19. Grol R, Grimshaw J. From best evidence to best practice: effective implementation of change in patients' care. Lancet 2003;362:1225-30.

20. Prior M, Guerin M, Grimmer-Somers K. The effectiveness of clinical guideline implementation strategies-a synthesis of systematic review findings. J Eval Clin Pract 2008;14: 888-97.

21. Boaz A, Baeza J, Fraser A, et al. Effective implementation of research into practice: an overview of systematic reviews of the health literature. BMC Res Notes 2011;4:212. 
22. Moher D, Liberati A, Tetzlaff J, et al. Preferred reporting items for systematic reviews and meta-analyses: the PRISMA statement. $J$ Clin Epidemiol 2009;62:1006-12.

23. Bero LA, Grilli R, Grimshaw JM, et al. Closing the gap between research and practice: an overview of systematic reviews of interventions to promote the implementation of research findings. BMJ 1998;317:465-8.

24. Read C, Kleiner B. Which training methods are effective? Manag Dev Rev 1996;9:24-9.

25. Forsetlund L, Bjørndal A, Rashidian A, et al. Continuing education meetings and workshops: effects on professional practice and health care outcomes. Cochrane Database Syst Rev 2009;(2):CD003030.

26. Giguère $A$, Légaré $F$, Grimshaw $J$, et al. Printed educational materials: effects on professional practice and healthcare outcomes. Cochrane Database Syst Rev 2012;(10):CD004398.

27. Chaix-Couturier C, Durand-Zaleski I, Jolly D, et al. Effects of financial incentives on medical practice: results from a systematic review of the literature and methodological issues. Int J Qual Health Care 2000;12:133-42.

28. Rohrbach LA, Grana R, Sussman S, et al. Type II translation: transporting prevention interventions from research to real-world settings. Eval Health Prof 2006;29:302-33.

29. Walker J, Postma K, McHugh GS, et al. Performance of the Hospital Anxiety and Depression Scale as a screening tool for major depressive disorder in cancer patients. J Psychosom Res 2007;63:83-91.
30. Ware JE Jr, Sherbourne CD. The MOS 36-item short-form health survey (SF-36): I. Conceptual framework and item selection. Med Care 1992;30:473-83.

31. Kessler RC, Barker PR, Colpe LJ, et al. Screening for serious mental illness in the general population. Arch Gen Psychiatry 2003;60:184-9.

32. Botti $\mathrm{M}$, Endacott $\mathrm{R}$, Watts $\mathrm{R}$, et al. Barriers in providing psychosocial support for patients with cancer. Cancer Nurs 2006;29:309-16.

33. Ostuzzi G, Matcham F, Dauchy S, et al. Antidepressants for the treatment of depression in patients with cancer. Cochrane Database Syst Rev 2015;6:CD011006.

34. Vodermaier A, Linden W, Siu C. Screening for emotional distress in cancer patients: a systematic review of assessment instruments. J Natl Cancer Inst 2009;101:1464-88.

35. Akbari A, Mayhew A, Al-Alawi MA, et al. Interventions to improve outpatient referrals from primary care to secondary care. Cochrane Database Syst Rev 2008;(4):CD005471. http://onlinelibrary.wiley.com/.

36. Bower P, Knowles S, Coventry PA, et al. Counselling for mental health and psychosocial problems in primary care. Cochrane Database Syst Rev 2011;(9):CD001025. http://onlinelibrary.wiley.com/.

37. Higgins JP, Green S. Cochrane handbook for systematic reviews of interventions. Wiley Online Library, 2008.

38. Tuinman MA, Gazendam-Donofrio SM, Hoekstra-Weebers JE. Screening and referral for psychosocial distress in oncologic practice. Cancer 2008;113:870-8. 